\title{
Investigation of the underling mechanism of ketamine for antidepressant effects in treatment-refractory affective disorders via molecular profile analysis
}

\author{
JUN QIAO $^{1 *}$, YUAN SUN $^{2 *}$, JINFANG WU $^{3}$ and LI WANG $^{2}$ \\ Departments of ${ }^{1}$ Psychiatry, ${ }^{2}$ Anesthesiology and ${ }^{3}$ Anesthesia Surgery, \\ The First Hospital of Hebei Medical University, Shijiazhuang, Hebei 050000, P.R. China
}

Received February 27, 2018; Accepted January 24, 2019

DOI: $10.3892 / \mathrm{etm} .2019 .7633$

\begin{abstract}
Ketamine elicits a rapid antidepressant effect in treatment-refractory affective disorders. The aim of the present study was to elucidate the underlying mechanism of this effect and to identify potential targets of ketamine for antidepressant effects. GSE73798 and GSE73799 datasets were downloaded from the Gene Expression Omnibus database. Differentially expressed genes (DEGs) were identified in hippocampus or striatum samples treated with ketamine, phencyclidyne or memantine compared with a saline or normal group at 1,2,4 and $8 \mathrm{~h}$. The overlapping DEGs were the DEGs in both hippocampus and striatum samples. Kyoto Encyclopedia of Genes and Genomes and BioCyc databases were used to perform functional annotation and pathway analyses. Protein-protein interactions (PPIs) were predicted using Search Tool for the Retrieval of Interacting Genes/Proteins version 9.1 for the DEGs in the striatum samples treated with ketamine, phencyclidine or memantine compared with normal samples. Reverse transcription-quantitative polymerase chain reaction was performed to determine mRNA levels. Perilipin 4 (Plin4), serum/glucocorticoid regulated kinase 1 ( $S g k 1)$, kruppel like factor 2 (Klf2) and DDB1 and CUL4 associated factor 12 like 1 (Dcaf12l1) were the overlapping DEGs in the striatum samples treated with the three drugs at different time points. The mRNA expression levels of Plin4, Sgkl and Klf2 were significantly higher $(\mathrm{P}<0.05)$, and the mRNA expression level of Dcaf12l1 was significantly lower in the striatum samples of the ketamine-treated group compared with the control group in an in vivo experiment. Both $S g k 1$ and $K l f 2$ were enriched in the 'forkhead box O (FoxO) signaling pathway', and $S g k l$ was
\end{abstract}

Correspondence to: Dr Li Wang, Department of Anesthesiology, The First Hospital of Hebei Medical University, 89 Donggang Road, Shijiazhuang, Hebei 050000, P.R. China

E-mail: liwang06@yeah.net

*Contributed equally

Key words: ketamine, antidepressant effect, molecular profile additionally enriched in the 'mechanistic target of rapamycin kinase (mTOR) signaling pathway'. PPI networks of DEGs in the striatum samples treated with ketamine, phencyclidine and memantine compared with normal samples were constructed, and Klf 2 was involved in more pairs and was therefore a gene hub in the three networks. The four genes, Plin 4, Sgkl, Klf2 and Dcaf12l1, were differentially expressed in all of the groups that treated with the three drugs and their expression levels were verified in in vivo experiments. The FoxO and mTOR signaling pathways may be involved in the underlying mechanism of the antidepressant effects of ketamine, and Plin4, Sgk1, Klf2 and Dcaf12ll may be potential biomarkers for depression in N-methyl-D-aspartic acid receptor antagonist treatment.

\section{Introduction}

Depression is a state of low mood and aversion to activity that can affect a person's thoughts, behavior, feelings and sense of well-being (1). It has been predicted that by 2030 , depression will account for the highest level of disability among all physical and mental disorders worldwide (2). People with depression may feel sad, anxious, empty, hopeless, helpless, dejected or worthless (3). Certain life events or changes may contribute to depression, including childbirth, menopause, financial difficulties, stress, social isolation or relationship difficulties (4). Adolescents are especially prone to experiencing depression following social rejection, peer pressure and bullying (5). In addition, several diseases (including hypothyroidism, multiple sclerosis, Parkinson's disease and chronic pain), and drugs (including heroin, intoxication, hallucinogens and inhalants) can cause or exacerbate depression $(6,7)$. Depression is also a symptom of treatment-refractory affective disorders (8). As indicated in the aforementioned studies, depression may be a temporary reaction to life events, a symptom of a medical condition, or a side effect of certain drugs or medical treatments.

N-methyl-D-aspartic acid (NMDA) receptor antagonists are a class of anesthetics that inhibit the action of NMDA receptors. Ketamine, phencyclidine and memantine are three common drugs belonging to the class of NMDA receptor antagonists $(9,10)$. They act primarily on the nervous system, with mild stimulant effects at subanesthetic doses, and effects of dissociation and hallucinations at higher doses (11). 
Ketamine is the primary anesthetic for burn victims and emergency patients with unknown medical history due to its moderate inhibition of respiration and circulation $(12,13)$. Certain studies have indicated that NMDA antagonists also exert antidepressant effects, particularly ketamine (14-16). However, the underlying molecular mechanism of this remains unclear. Therefore, the aim of the current study was to explore the underlying mechanism, and identify potential targets of ketamine for antidepressant effects based on molecular profile analysis.

\section{Materials and methods}

Expression profiles. mRNA expression profiles GSE73798 and GSE73799 were downloaded from the publicly available Gene Expression Omnibus (GEO) database (www.ncbi.nlm.nih. gov/geo). The GSE73798 profile contained data derived from 60 mouse hippocampus samples treated with ketamine, phencyclidyne, memantine and physiological saline or no treatment assessed at four time points (1, 2, 4 and $8 \mathrm{~h}$ after treatment). The GSE73799 profile included data derived from 60 mouse striatum samples that underwent the same treatments and assessments. Samples had been evaluated with the Illumina MouseWG-6 version 2.0 Expression BeadChip platform (Illumina, Inc., San Diego, CA, USA). A preliminary study using mice failed to establish and investigate the model of depression, and, therefore, a rat model was used in the present study to confirm differential gene expression.

Data processing and differentially expressed gene (DEG) analysis. Raw data were obtained and normalized with preprocessCore package (version 1.32.0; www.bioconductor. org/packages/3.2/bioc/html/preprocessCore.html), and probe symbols were converted to gene symbols. Subsequently, DEGs were identified in hippocampus or striatum samples individually treated with the three drugs, saline or no treatment and assessed at 1, 2, 4 and $8 \mathrm{~h}$, respectively. Each sample was analyzed three times. $\mathrm{P}<0.05$ and $\mid \log ($ fold-change $) \mid>0.05$ were used as the threshold criteria. A total of 48 sets of DEGs were obtained. Overlapping DEGs in the 24 sets were screened out for subsequent evaluation.

Functional annotation and pathway analysis. Kyoto Encyclopedia of Genes and Genomes (KEGG release 82; www.genome.jp/kegg) and BioCyc (version 21.0; biocyc.org) databases were used to perform functional annotation and pathway analysis for the overlapping DEGs. Gene ontology (GO) terms and pathway terms were selected at $\mathrm{P}<0.05$.

Construction of the protein-protein interaction (PPI) network. From the results of screening DEGs, the authors found that the three drugs had a greater effect on gene expression in striatum samples, therefore striatum samples were selected for further study. The search tool for the retrieval of interacting genes/proteins (STRING; version 9.1; string-db.org) is a biological database and web resource of known and predicted PPIs. In the current study, PPIs with a confidence score $>0.4$ were selected using STRING for the DEGs in the striatum samples treated with ketamine, phencyclidyne, memantine compared with normal samples. The PPI network was constructed using Cytoscape software (version 3.5.1; www. cytoscape.org/download.php).

Verification of associated genes. A total of 10 male Sprague-Dawley rats (age, 12 weeks; weight, 300-350 g) were purchased from Beijing Vital River Laboratory Animal Technology Co., Ltd. (Beijing, China). They were fed in specific-pathogen free (SPF) facilities under standard conditions and had access to food and water. The rats were housed at $18-29^{\circ} \mathrm{C}$ with a $12 \mathrm{~h}$ light/dark cycle and a relative humidity of 40-70\%; the rats had access to food and water ad libitum. A depression model was constructed using the chronic unpredictable mild stress method (17). The rats were randomly divided into a ketamine group and a control group, with 5 rats in each group. Ketamine $(25 \mathrm{mg} / \mathrm{kg}$; BOC Science Co., Ltd., Shirley, NY, USA) was injected intraperitoneally in the ketamine group, while a similar volume of sterile saline was injected intraperitoneally in the control group (18). At $8 \mathrm{~h}$ after drug administration, the rats were sacrificed via cervical vertebrae dislocation after anesthesia with pentobarbital sodium (45 mg/kg; intraperitoneal) and striatum samples were collected. All rat experiments were approved by the Animal Use and Care Committee of the First Hospital of Hebei Medical University (Shijiazhuang, China). Reverse transcription-quantitative polymerase chain reaction (RT-qPCR) (19) was performed to determine the mRNA expression levels of perilipin 4 (Plin4), serum/glucocorticoid regulated kinase 1 (Sgk1), kruppel like factor 2 (Klf2), and DDB1 and CUL4 associated factor 12 like 1 (Dcaf12l1) with the ABI Am1005 AgPath-ID ${ }^{\text {TM }}$ One-Step RT-PCR kit (Invitrogen; Thermo Fisher Scientific, Inc., Waltham, MA, USA) according to the manufacturer's protocol. The $2^{-\Delta \Delta \mathrm{Ct}}$ method (20) was used to quantify the results. The following primer sequences were used: Plin4 5'-GGGACAAGAACATGGGAAGC-3' (forward) and 5'-CCTTGACAAGACCTTTGGCC-3' (reverse); $S g k 1$ 5'-GAAGCTTGCCAACAACTCCT-3' (forward) and 5'-CGT GGGGATTTGAGGATGGA-3' (reverse); Klf2 5'-CTATCT TGCCGTCCTTTGCC-3' (forward) and 5'-GGCTCCGGG TAGTAGAACG-3' (reverse); Dcaf12ll 5'-CAGCAGCAA ACAGGTAGCAG-3' (forward) and 5'-CCTACCTCCCGA ACCTTCAG-3' (reverse). $\beta$-actin was as used as an internal reference, and the primer sequences were 5'-CTACAATGA GCTGCGTGTGG-3' (forward) and 5'-AGGCATACAGGG ACAACACA-3' (reverse).

Statistical analysis. SPSS software (version 17.0; SPSS, Inc., Chicago, IL, USA) was used for all statistical analyses and data were expressed as the mean \pm standard error of the mean. Student's t-test was used to compare groups. $\mathrm{P}<0.05$ was considered to indicate a statistically significant difference.

\section{Results}

DEGs. A total of 48 sets of DEGs were identified in hippocampus or striatum samples individually treated with the ketamine, phencyclidine or memantine, compared with the saline and normal groups at 1,2,4 and $8 \mathrm{~h}$. The gene numbers of the 48 sets of DEGs are presented in Table I. The overlapping genes were respectively identified in the three drug groups compared with both the saline and the normal group 
Table I. Gene numbers of the 48 sets of DEGs in hippocampus or striatum samples individually treated with ketamine, phencyclidine or memantine compared with the saline or normal group at 1,2,4 and $8 \mathrm{~h}$, respectively.

\begin{tabular}{llcccccccccc}
\hline & & \multicolumn{4}{c}{ Hippocampus } & \multicolumn{5}{c}{ Striatum } \\
\cline { 3 - 4 } Drug & Control & $1 \mathrm{~h}$ & $2 \mathrm{~h}$ & $4 \mathrm{~h}$ & $8 \mathrm{~h}$ & & $1 \mathrm{~h}$ & $2 \mathrm{~h}$ & $4 \mathrm{~h}$ & $8 \mathrm{~h}$ \\
\hline Ketamine & Saline & $13(2,11)$ & $7(6,1)$ & $2(2,0)$ & $0(0,0)$ & $113(23,90)$ & $122(30,92)$ & $235(121,114)$ & $103(41,62)$ \\
& Normal & $7(6,1)$ & $12(5,7)$ & $8(8,0)$ & $2(1,1)$ & $50(36,14)$ & $91(47,44)$ & $64(47,17)$ & $26(23,3)$ \\
Phencyclidine & Saline & $10(3,7)$ & $4(4,0)$ & $4(1,3)$ & $0(0,0)$ & $87(46,41)$ & $106(18,88)$ & $243(119,124)$ & $98(44,53)$ \\
& Normal & $8(4,4)$ & $14(3,11)$ & $15(10,5)$ & $5(2,3)$ & $50(41,9)$ & $43(9,34)$ & $38(21,17)$ & $31(27,4)$ \\
Memantine & Saline & $4(4,0)$ & $18(16,2)$ & $11(6,5)$ & $1(0,1)$ & $104(13,91)$ & $99(31,68)$ & $520(241,279)$ & $89(44,45)$ \\
& Normal & $7(7,0)$ & $16(16,0)$ & $7(7,0)$ & $1(1,0)$ & $19(14,5)$ & $43(30,13)$ & $134(60,74)$ & $13(11,2)$ \\
\hline
\end{tabular}

Data are presented as the total number of DEGs (upregulated DEGs, downregulated DEGs). DEGs, differentially expressed genes.

Table II. Gene numbers of the overlapping genes in three drug groups compared with both the saline and normal groups.

\begin{tabular}{lccccccccc}
\hline & \multicolumn{4}{c}{ Hippocampus } & & \multicolumn{3}{c}{ Striatum } \\
\cline { 2 - 3 } Drug & $1 \mathrm{~h}$ & $2 \mathrm{~h}$ & $4 \mathrm{~h}$ & $8 \mathrm{~h}$ & & $1 \mathrm{~h}$ & $2 \mathrm{~h}$ & $4 \mathrm{~h}$ & $8 \mathrm{~h}$ \\
\hline Ketamine & $3(2,1)$ & $1(0,1)$ & $2(2,0)$ & $0(0,0)$ & & $10(7,3)$ & $26(19,7)$ & $7(5,2)$ & $6(6,0)$ \\
Phencyclidine & $4(2,2)$ & $0(0,0)$ & $4(1,3)$ & $0(0,0)$ & & $20(18,2)$ & $11(3,8)$ & $2(1,1)$ & $9(7,2)$ \\
Memantine & $4(4,0)$ & $9(9,0)$ & $5(5,0)$ & $0(0,0)$ & & $5(4,1)$ & $14(10,4)$ & $40(18,22)$ & $2(1,1)$ \\
\hline
\end{tabular}

at different time points, and the gene numbers are presented in Table II. Furthermore, the overlapping genes were screened out at the four time points, and are presented in Table III. Plin4 was the only overlapping gene among the aforementioned 48 sets of DEGs, and Plin4, Sgk1, Klf2 and Dcaf12l1 were overlapping in the striatum samples treated with the three drugs at the different time points (Table III). Furthermore, Plin4, SgkI and $K l f 2$ were upregulated in the striatum samples treated with the three drugs compared with saline or no treatment, and Dcaf12ll was downregulated.

The results of RT-qPCR are presented in Fig. 1. The mRNA expression levels of Plin4, Sgk1 and Klf2 were significantly higher in the striatum samples of the ketamine group compared with the control group $(\mathrm{P}<0.05)$, while the mRNA expression of Dcaf12l1 was significantly lower compared with the control group $(\mathrm{P}<0.05)$. In addition, the expression of Plin4 in the hippocampus and striatum following treatment with the three drugs is presented in Figs. 2 and 3, respectively. The results indicated that the expression of Plin 4 was highest both in the hippocampus and striatum after treatment with ketamine compared with phencyclidine and memantine.

GO terms and pathway analysis. The enriched GO terms and KEGG pathways of Plin4, Sgk1, Klf2 and Dcaf12l1 were selected, and the results are presented in Tables IV and V. Plin4 was enriched in 1 KEGG pathway; $S g k 1$ was enriched in 5 GO terms and 4 KEGG pathways; and Klf2 was enriched in 4 GO terms and 3 KEGG pathways. Among these, Sgk1 and $K l f 2$ were enriched in the 'forkhead box O (FoxO) signaling pathway', and $S g k l$ was additionally enriched in the

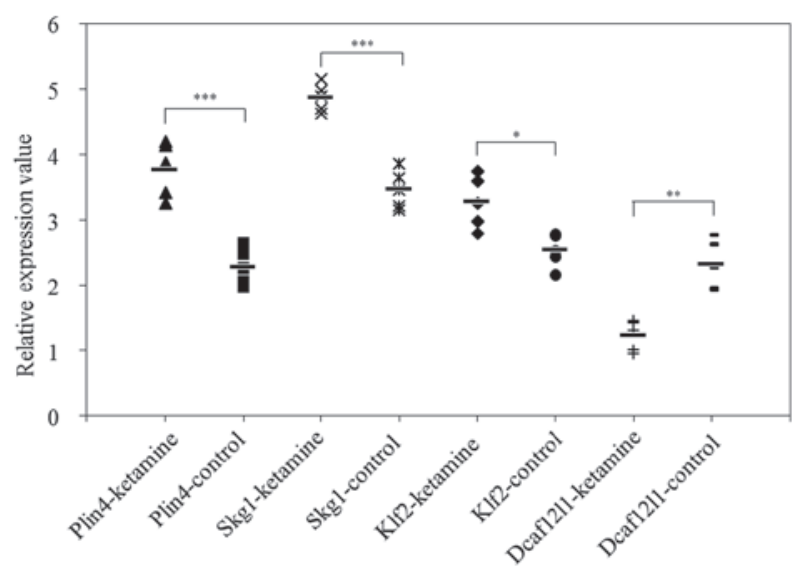

Figure 1. Relative expression levels of Plin4, Skgl, Klf2 and Dcaf12l1 in the striatum samples. ${ }^{* * *} \mathrm{P}<0.001,{ }^{* *} \mathrm{P}<0.01$ and ${ }^{*} \mathrm{P}<0.05$ as indicated. -ketamine, ketamine-treated striatum samples; -control, saline-treated striatum samples; SGK1, serum/glucocorticoid regulated kinase 1; Plin4, perilipin 4; Klf2, Kruppel like factor 2; Dcaf1211, DDB1 and CUL4 associated factor 12 like 1.

'mechanistic target of rapamycin kinase (mTOR) signaling pathway'. Plin4 and Dcaf12ll were enriched in no GO terms, and Dcaf12ll was enriched in no pathways.

PPI network. The PPI networks of DEGs in the striatum samples treated with ketamine, phencyclidine and memantine compared with normal samples are presented in Figs. 4-6, respectively. The results indicated that Klf2 was involved in more PPI pairs in these three PPI networks and was therefore the hub gene. 
Table III. Overlapping genes at the four time points.

\begin{tabular}{|c|c|c|c|}
\hline Tissue & Drug & Upregulated & Downregulated \\
\hline \multirow[t]{3}{*}{ Hippocampus } & Ketamine & Plin4, Sgk1, Gh, Txnip & Ttr \\
\hline & Phencyclidine & Klf2, Plin4, mtDNA_ND6 & $\begin{array}{l}m t D N A \_N D 4 L, S t k 32 c, \text { Hebpl, } m t-N d 4 l \text {, } \\
\operatorname{Prr} 7\end{array}$ \\
\hline & Memantine & $\begin{array}{l}\text { Plin4, Klf2, Gadd45g, Mfsd2a, Ddit4, } \\
\text { Cdkn1a, LOC240672, Dusp1, Sult1a1, } \\
\text { Sgk1, Fos, Egr4, Txnip, Tsc } 22 d 3\end{array}$ & \\
\hline \multirow[t]{4}{*}{ Striatum } & Ketamine & $\begin{array}{l}\text { Plin4, Sgk1, Klf2, Il22ra1, Vmn1r78, Slc13a4, } \\
\text { D7Zem2, Txnip, Ddit4, Olfr938, Kcne2, Star, } \\
\text { 1500015O10Rik, Tmem26, Wfdc2, Spink8, } \\
\text { Calml4, Olfr247, LOC380910, Ttr, Sostdc1, } \\
\text { Cap2, Gm4758, Cox8b, Vmn1r89, Lbp, } \\
\text { Col8a1, 932418N15Rik, Tmem28, Prr32, } \\
\text { LOC672705, Folr1, Otx2, Aqp1, Clic6, Wdr86 }\end{array}$ & $\begin{array}{l}\text { Dcaf12l1, Meox1, Olfr837, Slc6a13, } \\
\text { Crh, LOC100046930, Fut1, Krt31, } \\
\text { Fam171a1, Olfr938, Olfr1168, Fam } 214 b\end{array}$ \\
\hline & Phencyclidine & $\begin{array}{l}\text { Plin4, Sgk1, Klf2, Kl, Enpp2, Olfr474, Dctn1, } \\
\text { A030006E20Rik, D7Zem2, Aqp1, Fgf15, Cox8b, } \\
\text { Wfdc2, Zg16, Folr1, LOC381105, Hbb-b2, Wdr86, } \\
\text { Slc13a4, Ptgds, Crispld1, Osgin2, LOC383036, } \\
\text { Igfbp2, Cesla, Ctgf }\end{array}$ & $\begin{array}{l}\text { Dcaf12l1, Aldh1a1, Fbxw15, Egr2, } \\
\text { Cyp2c55, Egr4, Cldn5, Prr7, } \\
\text { A230065H16Rik, LOC194360, Cic, } \\
\text { Ethe1, Olfr1317 }\end{array}$ \\
\hline & Memantine & $\begin{array}{l}\text { Plin4, Sgk1, Klf2, Nfkbia, E130102H24Rik, } \\
\text { Cyp2c39, Pnpla2, Slc2a1, Gjb6, Gadd45g, } \\
\text { Il22ra1, Megf9, Cdkn1a, Nostrin, BAI3, } \\
\text { Ism1, Cfp, Ramp2, Txnip, Bcl6, Sult1a1, } \\
\text { Tsc22d3, LOC100044968, 9930108006Rik, } \\
\text { Adipor2, Thbd, Ddit4 }\end{array}$ & $\begin{array}{l}\text { Dcaf12l1, LOC331511, Cyp2c55, } \\
\text { Phyhip, LOC386233, Mid1ip1, } \\
\text { Rpl27a-ps1,LOC381105, Cplx2, Calm3, } \\
\text { Fam168b, Actb, Krt31, LOC } 241621, \\
\text { Pimreg, }\end{array}$ \\
\hline & & $\begin{array}{l}\text { Megf9, Cdkn1a, Nostrin, BAI3, Ism1, Cfp, } \\
\text { Ramp2, Txnip, Bcl6, Sult1a1, Tsc22d3, } \\
\text { LOC100044968, 9930108006Rik, Adipor2, } \\
\text { Thbd, Ddit4 }\end{array}$ & $\begin{array}{l}\text { C330049H01Rik, LOC433476, Cldn5, } \\
\text { LOC385068, Olfr1362, Hes5, Tmsb10, } \\
\text { Rhbdd3, BC037112, LOC } 382092, \\
\text { LOC382237, LOC673501, LOC381132 }\end{array}$ \\
\hline
\end{tabular}

\section{Discussion}

Genetic factors may promote or even cause the occurrence of depression (21). A systematic review found that major histocompatibility complex, class I-related gene polymorphisms, and glutamate decarboxylase (GAD) genes (GADI and $G A D 2$ ) contributed to the development of depression (22). One study reported that 5-hydroxytryptamine receptor $2 \mathrm{~A}$ functional rs6311 polymorphism may modulate the severity of depression symptoms in children with autism spectrum disorder (23). Another study reported that glutamate ionotropic receptor kainate type subunit 4 variants were involved in treatment-resistant depression (24). Apoptosis regulator BCL2 was considered to serve a role in mediating the outcome of antidepressant treatment (24). In the current study, rat models of depression were selected to investigate the underlying mechanism of treatment with ketamine. The present study was primarily based on bioinformatics and a comparison between the ketamine and control groups in vivo further demonstrated the effect of ketamine during depression, and provided an additional method for exploring the mechanism of action of ketamine. In addition, the underlying mechanism requires further verification in animal models and human trials, however the current results provide a preliminary basis for these.

In the present study, Plin4, Sgk1, Klf2 and Dcaf12l1 were the overlapping DEGs in the striatum samples treated with three NMDA receptor antagonists at different time points. Furthermore, the expression levels of Plin4, Sgk1 and Klf2 increased in the striatum samples of rat models of depression following administration of ketamine, and the expression of Dcaf12l1 decreased compared with the control group. Plin4 protein coats lipid droplets in adipocytes to protect them from lipases $(25,26)$. Plin4 is associated with insulin resistance and obesity risk $(27,28)$. In the current study, Plin4 was identified as the only overlapping DEG (upregulated) in hippocampus or striatum samples individually treated with ketamine, phencyclidine or memantine compared with the saline or normal groups at 1, 2, 4 and $8 \mathrm{~h}$. SGK1 protein contributes to the regulation of discrete developmental stages and pathological conditions including hypertension, diabetic neuropathy, ischemia, trauma and neurodegenerative diseases (29). Anacker et al (30) identified SGK1 as a mediator of the effects of cortisol on neurogenesis and glucocorticoid receptor function, with particular relevance to stress and depression. KLF2 protein is implicated in lung development, embryonic erythropoiesis, 


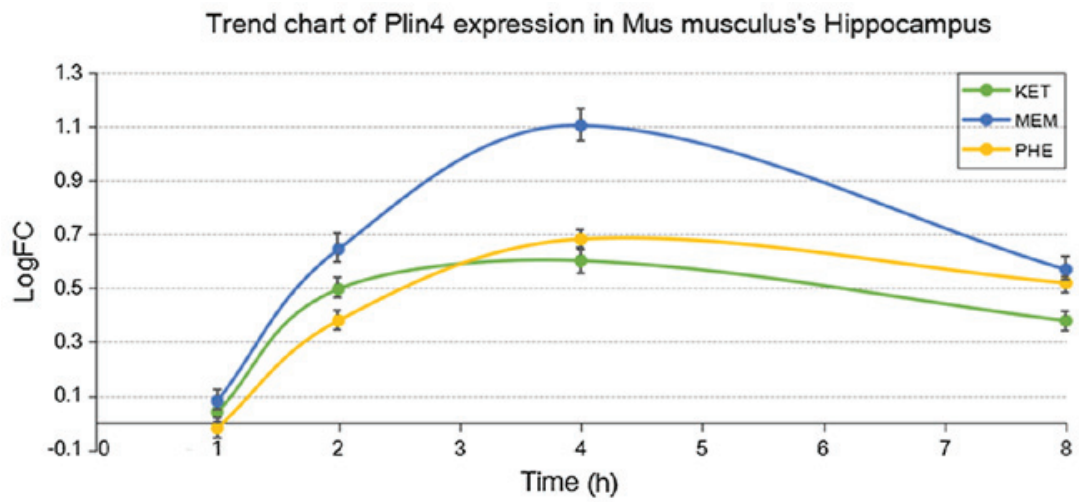

Figure 2. Expression of Plin4 in the hippocampus samples treated with KET, PHE and MEM. KET, ketamine; PHE, phencyclidine; MEM, memantine; Plin4, perilipin 4.

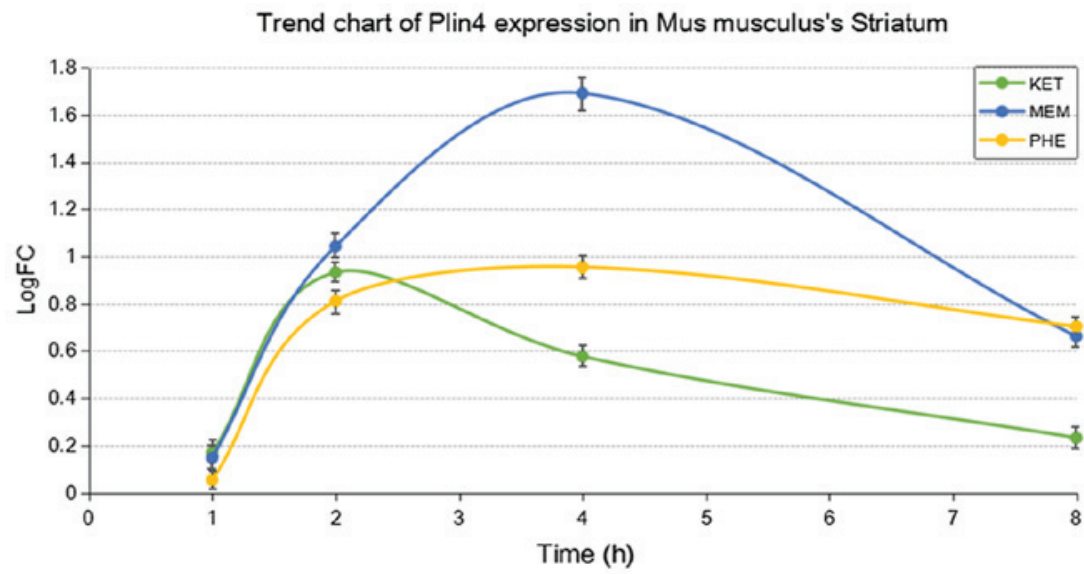

Figure 3. Expression of Plin4 in the striatum samples treated with KET, PHE and MEM. KET, ketamine; PHE, phencyclidine; MEM, memantine; Plin4, perilipin 4.

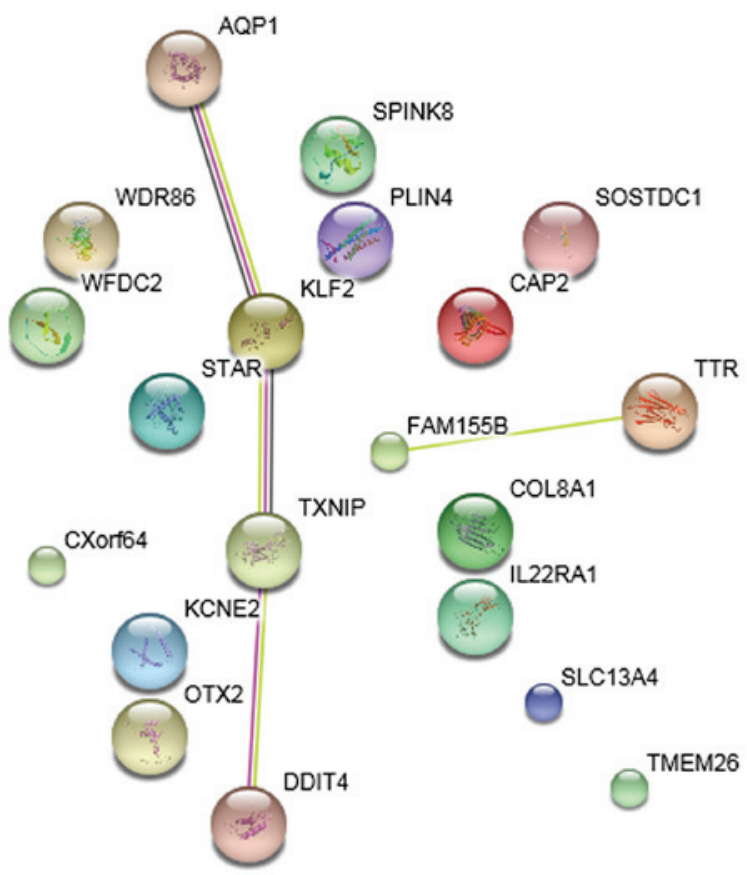

Figure 4. Protein-protein interaction network of differentially expressed genes in the striatum samples treated with ketamine. Small nodes represent proteins of unknown 3D structure, large nodes represent proteins with known or predicted 3D structure. Colored nodes represent query proteins and the first shell of interactors. Interactors with purple edges represent known experimentally determined interactions, brilliant green edges represent textmining interactions and dark edges represent co-expression interactions. STRING, search tool for the retrieval of interacting genes/proteins. 
Table IV. mRNA levels of Plin4, Sgkl, Klf2 and Dcaf12ll in the striatum samples.

Relative expression value

\begin{tabular}{lcccc}
\cline { 2 - 4 } Group & Plin4 & Sgk1 & Klf2 & Dcaf12l1 \\
\hline Ketamine group & $3.77 \pm 0.38$ & $4.87 \pm 0.19$ & $3.27 \pm 0.36$ & $1.23 \pm 0.21$ \\
Control group & $2.28 \pm 0.25$ & $3.46 \pm 0.27$ & $2.53 \pm 0.23$ & $2.31 \pm 0.34$ \\
P-value & $<0.0001$ & $<0.0001$ & 0.0402 & 0.0018 \\
T-value & 8.76 & 12.81 & -3.68 & 7.87 \\
\hline
\end{tabular}

Table V. The enriched GO terms of $S g k 1$ and Klf2.

\begin{tabular}{|c|c|c|}
\hline Gene & GO term & Function \\
\hline \multirow[t]{5}{*}{$S g k 1$} & GO:0004672 & 'Protein kinase activity' \\
\hline & GO:0004674 & $\begin{array}{l}\text { 'Protein serine/threonine } \\
\text { kinase activity' }\end{array}$ \\
\hline & GO:0005524 & 'ATP binding' \\
\hline & GO:0016301 & 'Kinase activity' \\
\hline & GO:0016740 & 'Transferase activity' \\
\hline \multirow[t]{4}{*}{ Klf2 } & GO:0003677 & 'DNA binding' \\
\hline & GO:0003700 & $\begin{array}{l}\text { 'Transcription factor activity, } \\
\text { sequence-specific DNA binding' }\end{array}$ \\
\hline & GO:0008270 & 'Zinc ion binding' \\
\hline & GO:0046872 & 'Metal ion binding' \\
\hline
\end{tabular}

GO, gene ontology; SGK1, serum/glucocorticoid regulated kinase 1; Klf2, Kruppel like factor 2.

epithelial integrity, T-cell viability and adipogenesis (31). Another study by Miller et al (32) was performed using ribosome-bound mRNA footprinting and deep sequencing, and confirmed that initiation of protein synthesis is a defining feature of antidepressant dose ketamine in mice; with the use of GO analysis, vasoactive intestinal peptide receptor 2 gene was identified as a potential target for antidepressant action. In the current study, Klf2 was involved in more pairs in the PPI network of DEGs in the striatum samples treated with ketamine, phencyclidine or memantine compared with normal samples. That was to say, Klf 2 was differentially expressed between the groups compared with the control, and that Klf2 exhibited the highest degree among all proteins in the PPI networks. However, to the best of the authors' knowledge, the association between Klf2 and depression or NMDA receptor antagonists has not been previously reported. Dcaf1211 protein is associated with embryonic development and idiopathic nonobstructive azoospermia $(33,34)$. The current study indicated that Plin4, Sgkl, Klf2 and Dcafl2ll were differentially expressed in depression models treated with ketamine, phencyclidine and memantine, which suggested that these genes may be the targets of the NMDA receptor antagonist treatment.

Duman et al (35) investigated the signaling pathway underlying the rapid antidepressant effects of ketamine, and the results demonstrated that the effects were associated
Table VI. Enriched KEGG pathways of Plin4, Sgkl and Klf2.

\begin{tabular}{lll}
\hline Gene & KEGG pathway & \multicolumn{1}{c}{ Name } \\
\hline Sgk1 & mmu04068 & 'FoxO signaling pathway' \\
& mmu04150 & 'mTOR signaling pathway' \\
& mmu04151 & 'PI3K-Akt signaling pathway' \\
& mmu04960 & 'Aldosterone-regulated sodium \\
& & reabsorption' \\
Plin4 & mmu03320 & 'PPAR signaling pathway' \\
Klf2 & mmu04068 & 'FoxO signaling pathway' \\
& mmu04371 & 'Apelin signaling pathway' \\
& mmu05418 & 'Fluid shear stress and \\
& & atherosclerosis' \\
\hline
\end{tabular}

KEGG, Kyoto Encyclopedia of Genes and Genomes; SGK1, serum/glucocorticoid regulated kinase 1; Plin4, perilipin 4; Klf2, Kruppel like factor 2.

with the stimulation of mTOR and increased expression levels of synaptic proteins. Li et al (36) observed that ketamine rapidly activated the mTOR pathway, leading to increased expression levels of synaptic signaling proteins and increased number and function of new spine synapses in the prefrontal cortex of rats, while inhibition of mTOR signaling completely blocked ketamine-mediated induction of synaptogenesis and behavioral responses in models of depression. The above results indicated that the effects of ketamine are opposite to the synaptic deficits that result from exposure to stress and could contribute to the rapid antidepressant effects of ketamine. The current study also found that $S g k l$, one of the key DEGs underlying the rapid antidepressant effects of ketamine (37), was enriched in the 'mTOR signaling pathway'. In addition, the present results indicated that both $S g k 1$ and $K l f 2$ were enriched in the 'FoxO signaling pathway'. FoxO is a subfamily of the fork head transcription factor family, serving roles in cell fate decisions (38). Polter et al (39) observed that FoxO may be a transcriptional target for treatment of anxiety and mood disorders, and serves a potential role in regulating mood-associated behavior. This study further indicated that mice displayed reduced anxiety when FoxO1 was knocked down in the brain (39). Hence, FoxO3a-deficient mice presented with an antidepressant-like behavior (39). The 


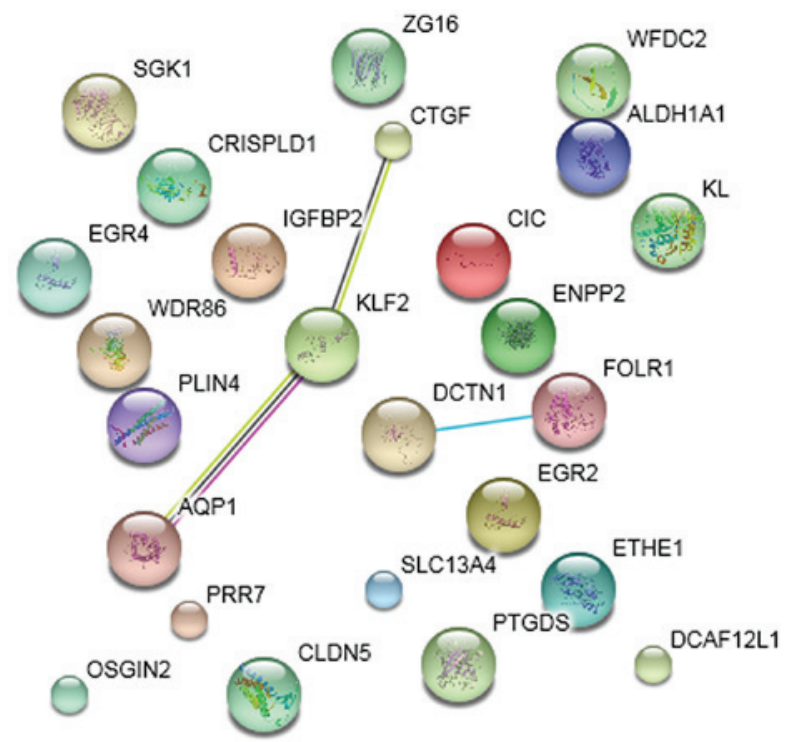

Figure 5. Protein-protein interaction network of differentially expressed genes in the striatum samples treated with phencyclidine. Small nodes represent proteins of unknown 3D structure, large nodes represent proteins with known or predicted 3D structure; colored nodes represent query proteins and first shell of interactors; interactors with light blue lines represent known interactions from STRING databases, purple edges represent known experimentally determined interactions, brilliant green edges represent textmining interactions, dark edges represent co-expression interactions. STRING, search tool for the retrieval of interacting genes/proteins.

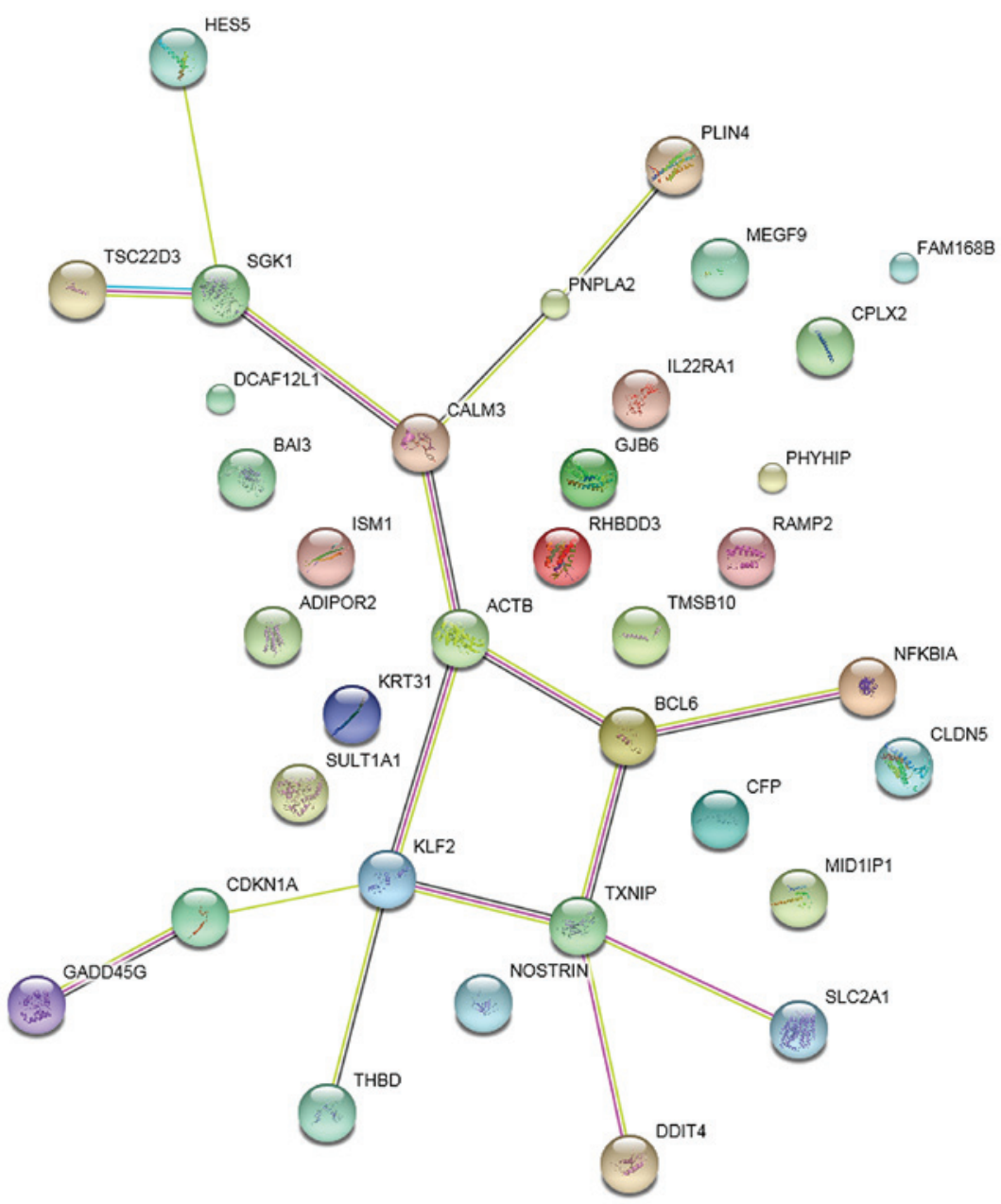

Figure 6. Protein-protein interaction network of differentially expressed genes in the striatum samples treated with memantine. Small nodes represent proteins of unknown 3D structure, large nodes represent proteins with known or predicted 3D structure; colored nodes represent query proteins and first shell of interactors; interactors with light blue lines represent known interactions from STRING databases, purple edges represent known experimentally determined interactions, brilliant green edges represent textmining interactions, dark edges represent co-expression interactions. STRING, search tool for the retrieval of interacting genes/proteins. 
FoxO signaling pathway is considered to be a therapeutic target in cancer $(40)$, and mediates stress responses $(41,42)$. Therefore, it is hypothesized that the mTOR and FoxO signaling pathways may be involved in the underlying mechanism of antidepressant effects of ketamine.

In conclusion, the present study suggested that the mTOR and FoxO signaling pathways may serve roles in the underlying mechanism of antidepressant effects of ketamine, and Plin4, Sgk1, Klf2 and Dcaf12l1 may be potential biomarkers for depression and targets for NMDA receptor antagonist treatment of depression.

\section{Acknowledgements}

Not applicable.

\section{Funding}

No funding received.

\section{Availability of data and materials}

All data generated or analyzed during the present study are included in this published article.

\section{Authors' contributions}

JQ and YS made substantial contributions to the conception and design of the study, and analysis of the data. JW interpreted data and drafted the manuscript. LW designed the study, drafted and revised the manuscript, gave final approval of the version to be published and agreed to be accountable for all aspects of the work.

\section{Ethics approval and consent to participate}

All rat experiments were approved by the Animal Use and Care Committee of the First Hospital of Hebei Medical University (Shijiazhuang, China).

\section{Patient consent for publication}

Not applicable.

\section{Competing interests}

The authors declare that they have no competing interests.

\section{References}

1. Saadat M, Behboodi ZM and Saadat E: Comparison of depression, anxiety, stress, and related factors among women and men with human immunodeficiency virus infection. J Hum Reprod Sci 8: 48-51, 2015.

2. HM, Vega WA, Williams DR, Tarraf W, West BT and Neighbors HW: Depression care in the United States: too little for too few. Arch Gen Psychiatry 67: 37-46, 2010.

3. Krishnan V and Nestler EJ: The molecular neurobiology of depression. Nature 455: 894-902, 2008.

4. Mata DA, Ramos MA, Bansal N, Khan R, Guille C Di Angelantonio E and Sen S: Prevalence of depression and depressive symptoms among resident physicians: A systematic review and meta-analysis. JAMA 314: 2373-2383, 2015.
5. Davey CG, Yücel M and Allen NB: The emergence of depression in adolescence: Development of the prefrontal cortex and the representation of reward. Neurosci Biobehav Rev 32: 1-19, 2008.

6. Saravane D, Feve B, Frances Y, Corruble E, Lancon C Chanson P, Maison P, Terra JL, Azorin JM and avec le soutien institutionnel du laboratoire Lilly: Drawing up guidelines for the attendance of physical health of patients with severe mental illness. Encéphale 35: 330-339, 2009 (In French).

7. Rogers D and Pies R: General medical with depression drugs associated. Psychiatry (Edgmont) 5: 28-41, 2008.

8. Al-Harbi KS: Treatment-resistant depression: therapeutic trends, challenges, and future directions. Patient Prefer Adherence 6: 369-388, 2012.

9. Aiyer R, Mehta N, Gungor S and Gulati A: A systematic review of NMDA receptor antagonists for treatment of neuropathic pain in clinical practice. Clin J Pain 34: 450-467, 2018.

10. Leung LS and Ma J: Medial septum modulates hippocampal gamma activity and prepulse inhibition in an, N-methyl-d-aspartate receptor antagonist model of schizophrenia. Schizophr Res 198: 36-44, 2018.

11. Rasmussen KG: Psychiatric side effects of ketamine in hospitalized medical patients administered subanesthetic doses for pain control. Acta Neuropsychiatr 26: 230-233, 2014.

12. Ceber $M$ and Salihoglu T: Ketamine may be the first choice for anesthesia in burn patients. J Burn Care Res 27: 760-762, 2006.

13. Heshmati F, Zeinali MB, Noroozinia H, Abbacivash R and Mahoori A: Use of ketamine in severe status asthmaticus in intensive care unit. Iran J Allergy Asthma Immunol 2: 175-180, 2003.

14. Maeng S, Zarate CA Jr, Du J, Schloesser RJ, McCammon J, Chen $G$ and Manji HK: Cellular mechanisms underlying the antidepressant effects of ketamine: Role of alpha-amino-3-hydroxy-5-methylisoxazole-4-propionic acid receptors. Biol Psychiatry 63: 349-352, 2008.

15. Murrough JW, Perez AM, Pillemer S, Stern J, Parides MK, aan het Rot M, Collins KA, Mathew SJ, Charney DS and Iosifescu DV: Rapid and longer-term antidepressant effects of repeated ketamine infusions in treatment-resistant major depression. Biol Psychiatry 74: 250-256, 2013.

16. Ardalan M, Rafati AH, Nyengaard JR and Wegener G: Rapid antidepressant effect of ketamine correlates with astroglial plasticity in the hippocampus. Br J Pharmacol 174: 483-492, 2017.

17. Jing XU: The establishment and evaluation of chronic unpredictable mild stress depression model. Chin J Behavioral Med Sci 12: 14-17, 2003 (In Chinese).

18. Wang J, Goffer Y, Xu D, Tukey DS, Shamir DB, Eberle SE, Zou AH, Blanck TJ and Ziff EB: A single subanesthetic dose of ketamine relieves depression-like behaviors induced by neuropathic pain in rats. Anesthesiology 115: 812-821, 2011.

19. Livak KJ and Schmittgen TD: Analysis of relative gene expression data using real-time quantitative PCR and the 2(-Delta Delta C (T)) method. Methods 25: 402-408, 2001.

20. Meijerink J, Mandigers C, van de Locht L, Tönnissen E, Goodsaid F and Raemaekers J: A novel method to compensate for different amplification efficiencies between patient DNA samples in quantitative Real-Time PCR. J Mol Diagn 3: 55-61, 2001.

21. Corrales E, Navarro A, Cuenca P and Campos D: Candidate gene study reveals DRD1 and DRD2 as putative interacting risk factors for youth depression. Psychiatry Res 244: 71-77, 2016.

22. Lacerda-Pinheiro SF, Pinheiro Junior RF, Pereira de Lima MA, Lima da Silva CG, Vieira dos Santos Mdo S, Teixeira Júnior AG, Lima de Oliveira PN, Ribeiro KD, Rolim-Neto ML and Bianco BA: Are there depression and anxiety genetic markers and mutations? A systematic review. J Affect Disord 168: 387-398, 2014.

23. Gadow KD, Smith RM and Pinsonneault JK: Serotonin 2A receptor gene (HTR2A) regulatory variants: Possible association with severity of depression symptoms in children with autism spectrum disorder. Cogn Behav Neurol 27: 107-116, 2014.

24. Milanesi E, Bonvicini C, Congiu C, Bortolomasi M, Gainelli G, Gennarelli $\mathrm{M}$ and Minelli A: The role of GRIK4 gene in treatment-resistant depression. Genet Res (Camb) 97: e14, 2015.

25. Wolins NE, Quaynor BK, Skinner JR, Schoenfish MJ, Tzekov A and Bickel PE: S3-12, Adipophilin, and TIP47 package lipid in adipocytes. J Biol Chem 280: 19146-19155, 2005.

26. Chen W, Chang B, Wu X, Li L, Sleeman M and Chan L: Inactivation of Plin4 downregulates Plin5 and reduces cardiac lipid accumulation in mice. Am J Physiol Endocrinol Metab 304: E770-E779, 2013. 
27. Peters SJ, Samjoo IA, Devries MC, Stevic I, Robertshaw HA and Tarnopolsky MA: Perilipin family (PLIN) proteins in human skeletal muscle: The effect of sex, obesity, and endurance training. Appl Physiol Nutr Metab 37: 724-735, 2012.

28. Soenen S, Mariman EC, Vogels N, Bouwman FG, den Hoed M, Brown L and Westerterp-Plantenga MS: Relationship between perilipin gene polymorphisms and body weight and body composition during weight loss and weight maintenance. Physio Behav 96: 723-728, 2009 .

29. Schoenebeck B, Bader V, Zhu XR, Schmitz B, Lübbert H and Stichel CC: Sgk1, a cell survival response in neurodegenerative diseases. Mol Cell Neurosci 30: 249-264, 2005.

30. Anacker C, Cattaneo A, Musaelyan K, Zunszain PA, Horowitz M, Molteni R, Luoni A, Calabrese F, Tansey K, Gennarelli M, et al: Role for the kinase SGK1 in stress, depression, and glucocorticoid effects on hippocampal neurogenesis. Proc Natl Acad Sci USA 110: 8708-8713, 2013.

31. Pearson R, Fleetwood J, Eaton S, Crossley M and Bao S: Kruppel-like transcription factors: A functional family. Int $\mathrm{J}$ Biochem Cell Biol 40: 1996-2001, 2008.

32. Miller OH, Grabole N, Wells I and Hall J: Genome-wide translating mRNA analysis following ketamine reveals novel targets for antidepressant treatment. BioRxiv 2018.

33. Ramasamy R, Ridgeway A, Lipshultz LI and Lamb DJ: Integrative DNA methylation and gene expression analysis identifies discoidin domain receptor 1 association with idiopathic nonobstructive azoospermia. Fertil Steril 102: 968-973 e3, 2014.

34. Gerovska D and Arauzo-Bravo MJ: Does mouse embryo primordial germ cell activation start before implantation as suggested by single-cell transcriptomics dynamics? Mol Hum Reprod 22: 208-225, 2016
35. Duman RS, Li N, Liu RJ, Duric V and Aghajanian G: Signaling pathways underlying the rapid antidepressant actions of ketamine. Neuropharmacology 62: 35-41, 2012.

36. Li N, Lee B, Liu RJ, Banasr M, Dwyer JM, Iwata M, Li XY, Aghajanian G and Duman RS: mTOR-dependent synapse formation underlies the rapid antidepressant effects of NMDA antagonists. Science 329: 959-964, 2010.

37. Ficek J, Zygmunt M, Piechota M, Hoinkis D, Rodriguez Parkitna J, Przewlocki R and Korostynski M: Molecular profile of dissociative drug ketamine in relation to its rapid antidepressant action. BMC Genomics 17: 362, 2016.

38. Farhan M, Wang H, Gaur U, Little PJ, Xu J and Zheng W: FOXO signaling pathways as therapeutic targets in cancer. Int J Biol Sci 13: 815-827, 2017.

39. Polter A, Yang S, Zmijewska AA, van Groen T, Paik JH, Depinho RA, Peng SL, Jope RS and Li X: Forkhead box, class O transcription factors in brain: Regulation and behavioral manifestation. Biol Psychiatry 65: 150-159, 2009.

40. Farhan M, Wang H, Gaur U, Little PJ, Xu J and Zheng W: FOXO signaling pathways as therapeutic targets in cancer. Int J Biol Sci 13: 815-827, 2017

41. Huang P, Zhou Z, Shi F, Shao G, Wang R, Wang J, Wang K and Ding W: Effects of the IGF-1/PTEN/Akt/FoxO signaling pathway on male reproduction in rats subjected to water immersion and restraint stress. Mol Med Rep 14: 5116-5124, 2016.

42. Liang B, Moussaif M, Kuan CJ, Gargus JJ and Sze JY: Serotonin targets the DAF-16/FOXO signaling pathway to modulate stress responses. Cell Metab 4: 429-440, 2006.

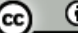

This work is licensed under a Creative Commons Attribution 4.0 International (CC BY 4.0) License. 